\title{
The Structural Connectivity Underpinning Language Aptitude, Working Memory, and IQ in the Perisylvian Language Network
}

\author{
Huadong Xiang \\ Donders Institute for Brain, Cognition and Behaviour
}

\section{Dan Dediu}

Donders Institute for Brain, Cognition and Behaviour and Max Planck Institute for Psycholinguistics

\section{Leah Roberts}

University of York and Max Planck Institute for Psycholinguistics

\section{Erik van Oort}

Donders Institute for Brain, Cognition and Behaviour

David G. Norris

Donders Institute for Brain, Cognition and Behaviour, Erwin L. Hahn Institute for Magnetic Resonance Imaging, and MIRA Institute for Biomedical Technology and Technical Medicine

\author{
Peter Hagoort \\ Donders Institute for Brain, Cognition and Behaviour and Max Planck Institute for \\ Psycholinguistics
}

In this article, we report the results of a study on the relationship between individual differences in language learning aptitude and the structural connectivity of language pathways in the adult brain, the first of its kind. We measured four components of language aptitude (vocabulary learning; sound recognition; sound-symbol correspondence; and grammatical inferencing) using the LLAMA language aptitude test. Spatial working memory, verbal working memory and $I Q$ were also measured as control factors.

Correspondence concerning this article should be sent to Leah Roberts, Centre for Language Learning Research, Department of Education, University of York, Heslington, York, YO10 5DD, UK. Internet: leah.roberts@york.ac.uk 
Diffusion Tensor Imaging was employed to investigate the structural connectivity of language pathways in the perisylvian language network. Regression analysis suggested significant correlations between most of these behavioural measures and the structural connectivity of certain language pathways, that is, grammatical inferencing and the BA45- and BA46-Temporal pathway, sound-symbol correspondence and the interhemispheric BA45 pathway, vocabulary learning and the BA47-Parietal pathway, IQ and the BA44- and BA-47Parietal pathways, the BA47-Temporal pathway and interhemispheric BA45 pathway, spatial working memory and the interhemispheric BA6 pathway and the BA47-Parietal pathway, and verbal working memory and the BA47-Temporal pathway. These findings provide further insights into the neural underpinnings of the variation in language aptitude of human adults and are discussed in relation to relevant findings in the literature.

\section{Introduction}

It is uncontroversial that adults differ enormously in their how successful they are at foreign language learning and one of the reasons behind this difference has been suggested to be variations in language learning aptitude (or talent) (see DeKeyser, 2000, 2012). Although the topic of language learning aptitude has been researched and discussed in great depth in the second language (L2) acquisition literature (see Sparks, 2012; DeKeyser, 2012; Robinson, 2005, for overviews), we know almost nothing about the neural underpinnings of the variation in language learning aptitude. One recent study has revealed a correlation between the structural connectivity of certain language pathways in the brain (the perisylvian language pathways connecting Broca's and Wernicke's territories) and performance in a language-learning related experimental task in adults (the Californian Verbal Learning Test, a test of word recall) (Catani et al., 2007). Specifically, the authors found that better performance on the semantic association task was associated with direct connections between the inferior frontal and the posterior temporal regions in the right hemisphere and interestingly, with bilateral representation of language networks, rather than extreme left-lateralization. This suggests an important role for the right hemisphere in word retrieval (see also Habib, Nyberg, \& Tulving, 2003), and an advantage for this type of semantic task for bilateral representation. Importantly for the current study, the fact that the Catani et al. demonstrated a heterogeneous pattern of lateralization of perisylvian pathways in their large sample (50) of healthy adults points to the need to examine individual variation in the structural connectivity of language pathways in the brain. In the study we report below, the focus on individual differences in performance on language aptitude tasks 
and their relationships with the structural connectivity of language pathways in the brain, an under-researched topic.

\section{Language Learning Aptitude}

Language aptitude and its association with foreign language learning success has been investigated for many years, mainly focusing on tutored learners and more recently also on learners in naturalistic settings (e.g., DeKeyser, 2000; Robinson, 2007). In the main, language learning aptitude is assumed to be a predominantly innate and somewhat fixed "gift" for learning languages; a cognitive capacity that some argue is largely independent of IQ, motivation and personality factors (e.g., Dörnyei \& Skehan, 2003). Most language learning aptitude tasks are based on the work of Carroll (e.g., Caroll, 1981; Carroll \& Sapon, 1959), and include tests of phoneme coding ability (establishing soundsymbol correspondences), grammatical sensitivity, word (semantic) learning and inductive learning ability (e.g., Modern Language Aptitude Test [MLAT]; Carroll \& Sapon, 1959; Defense Language Aptitude Battery [DLAB]; Peterson \& Al-Haik, 1976). This is also the case for the aptitude test used in the current study, the Llama Language Aptitude Test (LLAMA; Meara, 2005). Although not standardized, this test has been used with success in other language learning studies (e.g., Abrahamsson \& Hyltenstam, 2008), one of its strengths being that is language neutral (being based either on nonsense or extremely rare languages), as opposed to the majority of other aptitude tests which the participants must undertake in their first or dominant language. As well as measuring the participants' language learning aptitude, we also examined the impact of the control variables spatial working memory, verbal working memory and $I Q$, given that differences in successful second language learning may also be related to general cognitive abilities and skills in the first language (L1) (see Sparks, 2012).

\section{Mapping Brain Connectivity}

To study language pathways in the brain and their structural connectivity, Diffusion Tensor Imaging (DTI) was employed; a technique that can provide clear maps of brain connectivity (Figure 1). More specifically, DTI produces images of biological tissues weighted with the local micro structural characteristics of water diffusion. Therefore, using water molecules as a probe, the technique allows for the tracing of structural connections between brain regions in vivo, in a non-invasive manner and provides quantitative measures of brain white matter organization, offering a highly detailed picture of the architecture of brain tissue (Le Bihan et al., 2001). 


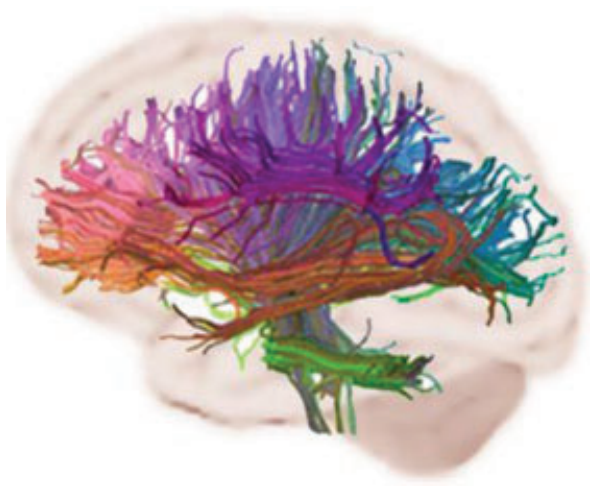

Figure 1 DTI (Diffusion Tensor Imaging) is capable of tracing structural connections between brain regions in vivo.

Below the results of the current study are presented, in which we examine the relationship between individual differences in performance on a battery language learning aptitude tests to the structural connectivity of language pathways in the brain.

\section{The Current Study}

\section{Method}

\section{Participants}

The data reported in the present study includes two samples. Sample 1 consisted of 15 participants ( 3 males) who had just graduated from high school. Sample 2 contained 20 participants ( 8 males) who were college/graduate students. The participants in both samples were healthy, right-handed adults between the ages of 19-26 years $(S D=1.78)$. All participants provided written informed consent and the study was conducted according to institutional guidelines of the local ethics committee (CMO protocol region Arnhem-Nijmegen, Netherlands). The majority of the participants were native speakers of German or Dutch, with English as a second language (see Appendix).

\section{Behavioral Tests}

\section{Language learning aptitude tests}

The LLAMA Language Aptitude Test (Meara, 2005) was used to gather data on the language learning aptitude of the participants. It includes four computerbased subtests which are designed to be independent of the first language (L1) of the test-taker. LLAMA_B is a vocabulary learning task, which measures the 
ability to learn reasonably large amounts of vocabulary in a relatively short time. LLAMA_D is a sound recognition task, which tests if participants can recognize short stretches of previously encountered spoken language. LLAMA_E is a sound-symbol correspondence task, which requires participants to work out the relationship between the sounds and the writing system based on previously heard syllables and their transliterations in an unfamiliar alphabet. Finally, LLAMA_F is a grammatical inferencing test that requires participants to work out the grammatical rules that operate in the unknown language.

Tests of general cognitive ability. Three more tests of general cognitive ability were carried out. A 20-minute version of the Raven Advanced Progressive Matrices (APM) Test was employed to measure $I Q .{ }^{1}$ This timed version has been shown to be an adequate predictor of the untimed APM score (Hamel \& Schmittmann, 2006). A new standard computerized version of reading span test was used to test verbal working memory (van den Noort, Bosch, Haverkort, \& Hugdahl, 2008). This version uses sentence materials and requires participants to read and attempt to understand sentences while remembering the final word. A spatial working memory test which includes one forward and one backward sub-test was also conducted. This test is a computerized adaptation based on the Spatial Span subtest of the Wechsler Memory Scale-Third Edition (Wechsler, 1997).

In order to understand the interrelationships between the various behavioral tasks, we performed a Principal Components Analysis (PCA). This procedure extracts a number of orthogonal components equal to the number of variables, with the first component (PC1) explaining most of the variance in the dataset, followed in decreasing order of the amount of variance explained by PC2, PC3, etc. Thus, by analysing the loadings of each of our tests on PC1, PC2, etc, we can better understand the nature of our tasks.

\section{DTI parameters}

Participants in both samples were scanned on a Siemens $3 \mathrm{~T}$ Trio scanner with the following parameters:

Sample 1: echo EPI (echo-planar imaging) sequence, repetition time $(\mathrm{TR})=10500 \mathrm{~ms}$, echo time $(\mathrm{TE})=94 \mathrm{~ms}, 110$ diffusion directions defined evenly across the sphere with a diffusion weighting of $b=1000 \mathrm{~s} / \mathrm{mm}^{2}$, 70 slices, matrix $=110 \times 110$, FOV $($ field of view $)=220 \times 220 \mathrm{~mm}^{2}$, isotropic voxels of $2.0 \mathrm{~mm}^{3}$

Sample 2: echo EPI sequence, $\mathrm{TR}=13000 \mathrm{~ms}, \mathrm{TE}=101 \mathrm{~ms}, 256$ directions at $\mathrm{b}=1500$ and 24 directions at $\mathrm{b}=0,70$ slices, matrix $=256 \times 256, \mathrm{FOV}=$ $256 \times 256 \mathrm{~mm}^{2}$, isotropic voxels of $2.0 \mathrm{~mm}^{3}$. 


\section{DTI data analysis}

DTI datasets were preprocessed by the DTI toolbox developed at the Donders Institute for Brain, Cognition and Behaviour (Zweier et al., 2009). The probabilistic tractography technique of the FMRIB Software Library (FSL) (Behrens et al., 2003) was employed to trace the possible pathways between the seed region in the left parietal lobe and each of the four subregions of Broca's complex (i.e., left BA6, BA44, BA45 and BA47, as the target region), respectively. To investigate the laterality of these pathways, the same tractography was applied to the right homologous regions. The possible pathways between posterior temporal lobe (seed) and each of the four subregions of Broca's complex (target) were traced in the same way. Seed regions were drawn based on our previous study on the topographical functional connectivity pattern of the perisylvian language network (Xiang, Fonteijn, Norris, \& Hagoort, 2010). Brain regions in the parietal lobe or posterior temporal lobe that showed significant functional connectivity to Broca's complex were taken as regions of interest (ROI). Voxels in these ROIs were then dilated (i.e., extended) with a radius of $4 \mathrm{~mm}$ under the restriction of not exceeding the boundaries of the parietal or posterior temporal lobe. The purpose of the dilation is to better accommodate the endpoints of fiber bundles. Target regions were defined according to the AAL (Automated Anatomical Labeling) template (Tzourio-Mazoyer et al., 2002). An exclusion mask of the sagittal midline was also implemented in the tractography to remove pathways that cross into the other hemisphere. The pathways were visualized with the help of MRIcron (http://www.cabiatl.com/mricro/mricron/index. html).

Two DTI measures were adopted to describe the structural connectivity properties of the traced pathways: the number of streamlines (Nstr) and the fractional anisotropy (FA). The probabilistic tractography repetitively samples from the distributions of voxel-wise principal diffusion directions, each time computing a streamline from the distribution on the location of the true fiber connections. Nstr counts how many such streamlines run from the predefined seed region to the predefined target region (Behrens et al., 2003). Nstr is an estimation of the number of real fibers based on the artificial reconstruction of these fibers. It should be kept in mind that Nstr (represented by a parameter called "waytotal" in FSL) is not a straightforward measure of tract integrity. It has been suggested that normalized values of waytotal should be used to avoid a possible tractability bias caused by subject motion or scanner noise. FA is a scalar measure of anisotropy. It is thought to reflect fiber density, axonal diameter, and myelination in white matter (Basser \& Pierpaoli, 1996). To avoid subjective thresholding, a probability-weighted average FA was calculated for 
each pathway and subject using the following equation (derived from: Hua et al., 2008):

$$
\text { Mean } \mathrm{FA}=\sum\left(\operatorname{Pr}_{\mathrm{i}}{ }^{*} \mathrm{FA}_{\mathrm{i}}\right) / \sum \operatorname{Pr}_{\mathrm{i}}
$$

Where $\operatorname{Pr}_{i}$ is the probability of the ith voxel to be part of the reconstructed tract, empirically decided by the number of streamlines that pass through the ith voxel divided by the total number of streamlines.

To assess the structural laterality of each connection, lateralization indices (LI) were calculated for both Nstr and FA using the following equation:

$$
\text { LI }=(\text { Left }- \text { Right }) /(\text { Left }+ \text { Right })
$$

To see whether the absolute difference between left and right connections also influence the behavioral measures, an absolute lateralization index (LA) was also calculated using the following equation:

$$
\text { LA }=\text { Left }- \text { Right }
$$

Thus for both LI and LA, a more positive value indicates "more leftlateralized" and a more negative value means "more right-lateralized."

Regression analysis on the relationship between behavioral measures and structural connectivity profiles

Stepwise regression analysis implemented in SPSS 16.0 (SPSS, Inc., Chicago $I L)$ was used to investigate whether each of the behavioral measures that were gathered could be explained by the connectivity profiles of certain pathways. Behavioral measures were regressed onto the following:

- Number of streamlines (Nstr)

- Lateralisation indeces of Nstr (LI_Nstr)

- Lateralisation index of Nstr (LA_Nstr)

- the summation of left and right Nstr (Sum_Nstr).

Sum_Nstr was included as a regressor to control for brain size and the bias of tractability caused by subject motion and scanner noise. The gender of the 
Table 1 Loadings of the behavioral measures on the principal components

\begin{tabular}{lccr}
\hline & \multicolumn{3}{c}{ Component } \\
\cline { 2 - 4 } & 1 & 2 & 3 \\
\hline Vocabulary learning & .657 & .214 & -.273 \\
Sound recognition & .438 & -.712 & -.108 \\
Sound-symbol correspondence & .618 & .386 & -.111 \\
Grammatical inferrencing & .832 & -.094 & -.328 \\
IQ & .341 & .667 & .119 \\
Spatial working memory & .349 & .116 & .816 \\
Verbal working memory & .587 & -.436 & .413 \\
\hline
\end{tabular}

subject (gender) and the sample to which the subject belongs (sample) were entered as control regressors as well.

The same stepwise regression analyses were carried out to investigate the relationship between the behavioral measures and the fractional anisotropy (FA).

\section{Results}

\section{PCA Analysis of the Behavioral Measures}

The first three Principal Components (PCs) accounted for $67 \%$ of the variance. The first PC (PC1) explains 32.6\% of the variance. The second PC (PC2) explains $19.4 \%$ and the third (PC3) $15 \%$ of the variance. The corresponding loadings are shown in Table 1.

PC1 has positive loadings on all 7 behavioral measures indicating that all these behavioral measures may be important for foreign language learning. PC2 had positive loadings on vocabulary learning, sound-symbol correspondence, spatial working memory, and $I Q$ (the largest loading) and negative loadings on sound recognition, verbal working memory and grammatical inferencing. This suggests that vocabulary learning, establishing sound-symbol correspondences and spatial working memory are more related to IQ than verbal working memory, the ability to recognise sounds, and deducing grammatical patterns in the input. PC3 distinguished those abilities assumed to relate specifically to language aptitude from more general cognitive abilities, with negative loadings on vocabulary learning, sound recognition, sound-symbol correspondence, grammatical inferencing and verbal working memory and positive loadings on $I Q$ and spatial working memory. 
a

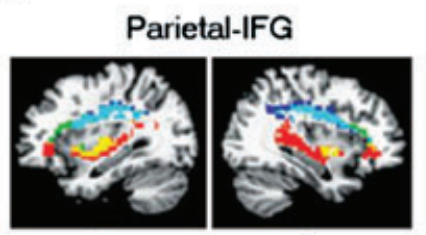

L

R

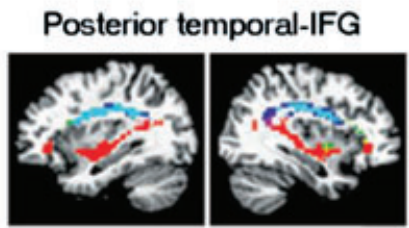

L

R

- Parietal (or posterior temporal)-BA44 [P1]

Parietal (or posterior temporal)-BA45 [P2]

Parietal (or posterior temporal)-BA47 [P3]

Overlap of [P1] \& [P2]

Overlap of $[\mathrm{P} 1]$ \& $[\mathrm{P} 3]$

b

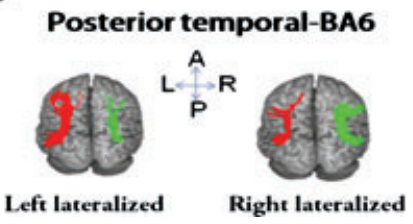

Figure 2 (a) Sagittal view of traced pathways between parietal/posterior temporal lobe and 3 subregions (BA44, 45 and 47) of IFG (Broca's complex). (b) Left or Right lateralized BA6-Temporal pathways projected on the MRICRON brain template. L: Left hemisphere; R: right hemisphere; A: anterior brain; P: posterior brain.

\section{Language Pathways}

DTI fiber tractography discovered robust structural connections for all seedtarget pairs in $97 \%$ of the participants (Figure 2). While connections between the parietal/posterior temporal lobe and BA6/BA44 are observed to lie mainly in the dorsal part of the brain, connections between the two lobes and BA47 go through the ventral part. Connections between the two lobes and BA45 are seen in both dorsal and ventral parts of the brain. Interhemispheric pathways were shown in Figure 3; all of these pathways go through the corpus callosum.

\section{The Relationship Between Behavioral Measures and Structural Connectivity in the Language Pathways}

The step-wise regression analyses found the most optimal models for vocabulary learning (for Nstr, adjusted $R^{2}=0.14, p=0.03$ ), sound recognition (for Nstr, adjusted $R^{2}=0.37, p=.04$ ), sound-symbol correspondence (for FA, adjusted $R^{2}=0.25, p=.003$ ), grammatical inferencing (for Nstr, adjusted $R^{2}=0.36, p=0.002$ ), $I Q$ (for Nstr, adjusted $R^{2}=0.76, p<.0001$; for FA, 


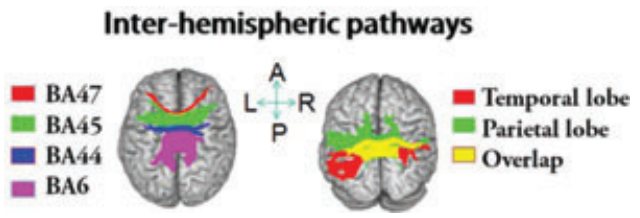

Figure 3 Interhemispheric pathways of BA6, BA44, BA45, BA47, parietal lobe and posterior temporal lobe projected on the MRICRON brain template. L: left hemisphere; $\mathrm{R}$ : right hemisphere; A: anterior brain; P: posterior brain.

adjusted $R^{2}=0.24, p=.005$ ), spatial working memory (for Nstr, adjusted $R^{2}=0.32, p=.002$; for FA, adjusted $R^{2}=0.23, \mathrm{p}=0.006$ ), and verbal working memory (for Nstr, adjusted $R^{2}=0.34, p=.003$ ). Table 2 shows the details of the best predictors suggested by each of these models.

It should be noted that the significance of the predictor of Nstr LA47P ( $a b$ solute lateralization index of BA47-parietal pathway) for vocabulary learning did not survive a Bonferroni correction. We still report this result because it is relevant to previous findings (see Discussion for more details). All the significant results reported are Bonferroni corrected unless otherwise specified. Figure 4 shows the correlations between the four language aptitude tests and their best predictors.

\section{Discussion}

\section{Behavioral Measures}

For the 7 behavioral measures, the first three Principal Components (PCs) explained most of the variance. PC1 was the largest component (32.6\%) and represents the agreement between all these behavioral measures, suggesting that a general ability might be important to L2 acquisition. PC2 explains $19.4 \%$ and seems to suggest that vocabulary learning, sound-symbol correspondence and spatial working memory are more closely related to general $I Q$ than sound recognition and verbal working memory. Finally, PC3 explains $15 \%$ of the variance and distinguishes the tasks specifically designed to tap into language aptitude (vocabulary learning, sound recognition, sound-symbol correspondence and grammatical inferencing) from more generic measures (IQ, spatial and verbal working memory).

\section{Anatomical Connections in the Perisylvian Language Network}

Our fiber tracking results are generally in agreement with previous findings on language pathways (Catani, Howard, Pajevic, \& Jones, 2002; Friederici, 
Table 2 Results of regression analysis on the relationship between behavioral scores and structural connectivity

Nstr $\quad$ FA

a. Vocabulary learning

\section{LA47P}

b. Sound recognition

Gender

c. Sound-symbol correspondence

Inter45

d. Grammatical inferencing

LI45T

Sum6T

e. $I Q$

Sample

LA44P

Inter 45

LA47P

$L I 47 T$

L47T

f. Spatial working memory

Inter6

LI47P

g. Verbal working memory

Sum $47 T$
$-.41^{! *}$

n.s.

$.46^{*}$

$.34^{! *}$

n.s.

$.72^{* *}$

$.51^{*}$

n.s.

$.51^{*}$

n.s.

$.68^{* *}$

$.49^{*}$

$-.56^{* *}$

n.s.

$-.32^{*}$

n.s.

$-37^{*}$

n.s.

$.38 *$

n.s.

n.s.

$-.44^{*}$

$.56^{* *}$

n.s.

n.s.

$-.56^{*}$

$-.48^{*}$

n.s.

*: Bonferroni corrected $p<.05 ;{ }^{* *}$ : Bonferroni corrected $p<.01 ;$ !*: Bonferroni $^{*}$ not corrected $p<.05$; n.s.: not significant; L: left; R: right; LI: lateralization index; LA: absolute difference between L and R; Inter: interhemisphere connectivity; Sum: summation of L and R; 6: BA6; 44: BA44; 45: BA45; 47: BA47; T: posterior temporal lobe; P: parietal lobe.

2009). The interhemisptheric pathways all go along the corpus callosum. The dorsal pathways (BA6, 44 and part of BA45-Parietal and Temporal pathways) in our results may well represent the four components of the superior longitudinal fasciculus (SLF) (Makris et al., 2005). SLF includes a superior portion consisting of horizontal fibers that connect the superior parietal lobe (SLF-I), the angular gyrus (SLF-II) and the supramarginal gyrus (SLF-III) to ipsilateral frontal and opercular areas. The inferior portion consists of long arched fibers that connect the superior and middle temporal gyri with frontal ipsilateral areas (SLF-IV). This inferior portion is also termed the arcuate fasciculus (AF). The 
a

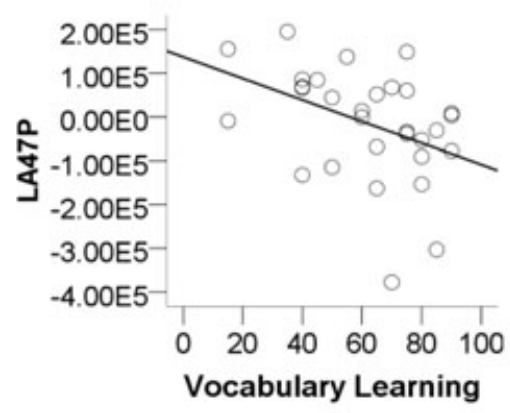

C

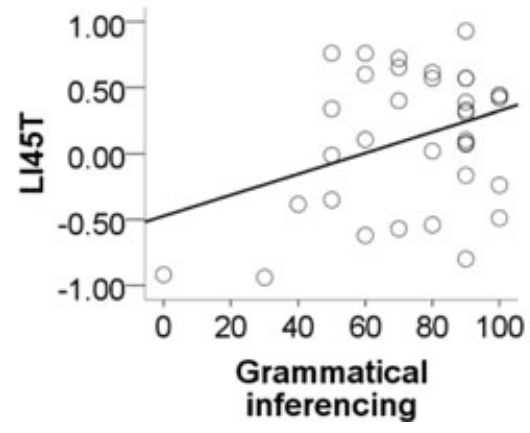

b

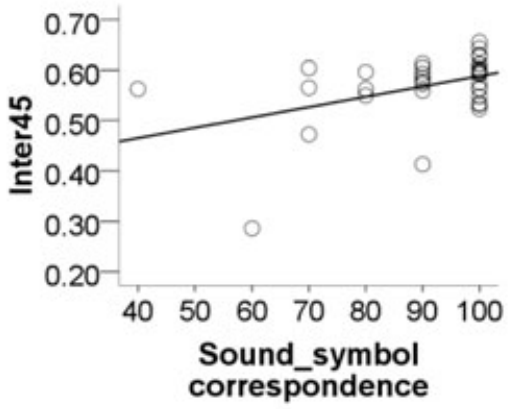

d

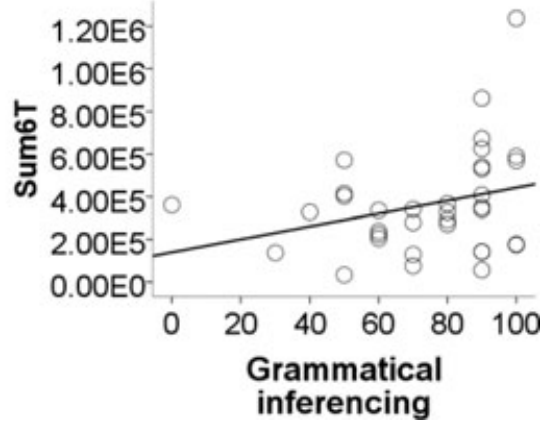

Figure 4 The correlation between a) the score of vocabulary learning test and the absolute lateralization index of Nstr (number of streamlines) of the BA47-Parietal pathway; b) the score of sound-symbol correspendence test and the FA of the interhemispheric BA45 pathway; c) the score of grammatical inferencing test and the lateralization index of FA of the BA45-Temporal pathway; d) the score of grammatical inferencing test and the summation of left and right Nstr of the BA6-Temporal pathway. LA: absolute lateralization index, i.e., absolute difference between the left and right pathway; LI: lateralization index; Inter: interhemispheric; Sum: summation of the left and right pathway; 6: BA6; 45: BA45; 47: BA47; T: posterior temporal lobe; P: parietal lobe.

dorsal temporal-frontal pathways we found are also proximate to the direct segmentation of AF described by Catani et al; the dorsal parietal-frontal pathways we found seem to correspond to the "anterior segment" of the indirect Broca-to-Wernicke pathway running parallel to the main AF described by Catani et al (2007). 
The ventral posterior temporal-frontal pathways (BA47 and part of BA45Temporal pathways) go along the extreme capsule, part of which connects the inferior frontal gyrus (IFG) and the middle-posterior portion of the superior temporal gyrus (STG) (Makris \& Pandya, 2009). The ventral pathways between parietal lobe and frontal lobe (i.e., BA47 and part of BA45-Parietal pathways in the present results) have been described in the dissection of the superior fronto-occipital fasciculus by Catani et al. (2002). The medial parts of the ventral parietal/posterior temporal-frontal pathways may also consist of fibers of the cingulum. The cingulum runs within the cingulated gyrus all around the corpus callosum. It contains fibers of different length. Its short U-shaped fibers connect the medial frontal, parietal, occipital, and temporal lobes and different portions of the cingulated cortex (Catani \& de Schotten, 2008).

\section{Language Aptitude Measures and Language Pathway Connections} Grammatical Inferencing and BA45-Temporal and BA6-Temporal Pathways The best predictors for Grammatical inferencing were LI45T and Sum6T. This finding sits well with other findings in the literature, specifically, that the function of BA45 for syntactic/grammatical processing (see, e.g., Bookheimer, 2002; Musso et al., 2003). The functional connection between left BA45 and the posterior temporal lobe has also been suggested to resemble the syntactic pathway in the perisylvian language network (Xiang, Fonteijn, Norris, \& Hagoort, 2010). Overall, this result confirms the supposition that the lateralization of BA45-temporal pathway is important for grammatical inferencing.

The Sum_6T predictor (the summation of Nstr left and right BA6-temporal pathway) for grammatical-inferencing also fits with other work that has found part of BA6 to be responsible for the processing of hierarchical structures in artificial grammar learning (Friederici, Bahlmann, \& Schubotz, 2008). Furthermore, in a recent study (Xiang et al., submitted), we found that the lateralization of BA6-Temporal pathway changed with increasing L2 proficiency, while the summation of Nstr of both left and right BA6-Temporal pathway remained relatively constant. This suggests that the summation of Nstr of left and right BA6-Temporal pathway could be a possible indicator of the neural substrate responsible for components of language aptitude. However, we note that this finding should be viewed with some caution, because the summation of Nstr was considered as a control regressor. Specifically, it may be subject to the influence of brain size (i.e., bigger brains may contain more fibers in total), and also to subject motion or scanner noise (i.e., motion and noise may influence the total number of fibers which can be traced in the brain). 


\section{Sound Recognition and Gender}

For sound recognition, gender was found to be the only significant predictor. T-tests also revealed a significant gender difference on sound recognition. Previous studies have shown that there is more variation in females than males when performing sound perception tasks, with performance in females being more affected by factors like voicing and context (Liederman et al., 2011; Westerhausen, Helland, Ofte \& Hugdahl, 2010; Yu, 2010). The larger variation on this task in females might lead to poorer performance in females compared to males. However, it should be noted that our sample comprised more than double the number of females (24) than males (11) (due to the difficulty of recruiting males in the target population), and so it is not possible to make any strong claims on the basis of this finding.

\section{Sound-Symbol Correspondence and Interhemispheric BA45 Pathway}

The best predictor found for sound-symbol correspondence was Inter45. The literature is rather sparse in this respect so we will try to present some hypotheses on the significance of this result. The Sound-symbol correspondence test requires participants to work out the relationship between sounds and the writing system based on previously heard syllables and their transliterations in an unfamiliar alphabet. It might resemble the link between phonology and semantics (as represented by abstract symbols in this case). Besides its function for syntactic processing, BA45 has been found to be an overlapping area for both phonological and semantic processing (Bookheimer, 2002; Poldrack et al., 1999; Xiang et al., 2010). This might tentatively suggest that BA45 could act as a possible neural link between phonology and semantics. The interhemispheric connectivity of BA45 thus could be a possible neural substrate for performing sound-symbol correspondences: a fruitful area for future investigation.

Vocabulary learning and BA47-parietal pathway. LA47P was suggested as the best predictor of vocabulary learning. Although this result did not survive Bonferroni correction, it seems to be in line with previous findings. The parietal lobe has been reported to be of great relevance for vocabulary knowledge (Mechelli et al., 2004; Price et al., 2007; Richardson \& Price, 2009). These structural imaging studies have consistently located an area in the parietal lobe (i.e., the supramarginal gyrus) that supports vocabulary learning. The grey matter density in this area was found to be positively correlated with vocabulary knowledge and was suggested to be "a neural marker for the number of words learnt" (Richardson \& Price, 2009, p. 514). BA47 is known to be a semantic/lexical region as suggested by a comprehensive meta-analysis of language studies (Bookheimer, 2002) and as proposed by the Memory, Unification and 
Control model of language processing (MUC, Hagoort, 2005; Xiang et al., 2010). Overall, the present results seem to suggest that the communication between BA47 and the parietal lobe is very important in explaining the individual differences in vocabulary learning, but more research is necessary to strengthen this claim.

\section{Control Variables and Language Pathways}

IQ And Sample, BA44-Parietal, Interhemispheric BA45, BA47-Parietal, BA47-Temporal Pathways

Several significant predictors were found for IQ: sample, LA44P, Inter45, $L A 47 P, L I 47 P$ and $L 47 T$. The neural substrate of $I Q$ has been shown to involve a distributed network including the frontal lobe (BA44, 45,47), temporal lobe and parietal lobe (Deary, Penke, \& Johnson, 2010; Jung \& Haier, 2007; Shaw, 2007). Previous DTI studies have also revealed that $I Q$ is correlated with the white matter integrity of both dorsal and ventral language pathways, such as the arcuate and uncinate fasciculi (Thompson et al., 2008). Our results fit with these findings. As for the factor of sample, it is unsurprising to have found it to be a predictor of $I Q$ in this study because participants in Sample 1 were middle school graduates, while participants in Sample 2 were college/graduate students. The result of the t-test confirmed that participants in Sample 2 indeed had significantly higher IQs than those in Sample 1.

\section{Spatial Working Memory and Interhemispheric BA6, BA47-Parietal Pathways} For spatial working memory, Inter6 and LI47P were found to be the best predictors. A recent meta analysis (Bullmore, Owen, McMillan, \& Laird, 2005) shows that spatial working memory tasks always involve BA6 and the Parietal lobe. The present results suggest that the interhemispheric connectivity of BA6 and the lateralization of Nstr BA47-parietal lobe are both important for spatial working memory, although it is not clear what the role of BA47 might be.

\section{Verbal Working Memory and BA47-Temporal Pathway}

For verbal working memory, Sum47T was found to be the best predictor. It should be mentioned that the present study used a different paradigm for testing the verbal working memory compared to many previous studies. We used sentence materials and asked participants to try to read and understand the sentence while remembering its last word. This paradigm may have led participants to rely on the meaning of the sentence to help them to remember the final words. This could be the reason why the semantic pathway (BA47-Temporal) 
was found as a predictor for verbal working memory in the present results. Previous studies have most often used simpler stimuli such as consonants, for which participants might mainly rely on a shallower processing of these stimuli, and these studies reported that BA6/44/45 and the dorsal phonological pathway were relevant for verbal working memory (Catani et al., 2007; D’Esposito et al., 1998).

\section{Caveats, and Future Directions}

This study investigated the relationship between individual differences in language learning aptitude, as measured by the LLAMA test, and the structural connectivity of language pathways in the adult brain. Significant correlations were found between the results of the four components of the aptitude tests and the structural connectivity of certain language pathways, that is, grammatical inferencing and the BA45- and BA46-Temporal pathways, sound-symbol correspondence and the interhemispheric BA45 pathway, vocabulary learning and the BA47-Parietal pathway. Significant correlations were also observed between the scores on the control measures and language pathways: $I Q$ and BA44- and BA47-Parietal pathway, BA47-Temporal pathway and the interhemispheric BA45 pathway, spatial working memory and the interhemispheric BA6 pathway and the BA47-Parietal pathway, and verbal working memory and the BA47-Temporal pathway. Although an exploratory study, the findings provide further insights into the neural underpinnings of the variation in the language aptitude. We do note, however, that some of the behavioral measures used in this study are far from being well-understood: as a prominent example, it is still unclear what exactly the Raven test measures and the relationships between this construct and second language learning. Similar uncertainties surround the other measures, especially the LLAMA test, which has not been yet standardized. Given these, our results should be further refined by future studies, but we think they provide a very interesting first finding.

An important caveat concerns the nature of our study: what we report here are correlations between behavioral measures of second language learning and (the change in) structural properties of the brain. However, to be able to draw any conclusion concerning possible causal relationships between these, we would need different experimental designs.

It should be noted that the regression analyses of Nstr and FA did not suggest the same predictors. This might indicate that they probably do not measure the same properties of a pathway, a phenomenon that has been previously reported in DTI studies (Catani et al., 2007; Lebel \& Beaulieu, 2009). 
Another caveat is that currently available methods for measuring the structural connectivity are subject to distortions due to factors like crossing fibers and limitations of the algorithms, which may result in a decreased statistical power of the present study. The DTI technique is based on the observed anisotropy of water self-diffusion in white matter, which is thought to be caused by the diffusion boundaries formed by the myelinated fiber bundles. DTI has been proven sufficient to infer the dominant fiber direction. But in cases of partial voluming as well as for crossing, touching, fanning or bending fiber configurations, the diffusion modelling may become distorted (Schultz \& Seidel, 2008). For example, measurement of FA in regions where there are crossing fibers are found vulnerable to noise and scanning parameters. The SD (standard deviation) differences of FA were found to correspond to roughly $5-7 \%$ of the trace value in these regions, and much lower in other regions (0-2\%) (Alexander, Hasan, Lazar, Tsuruda \& Parker, 2001). In the present study, we employed the advanced probabilistic tractography to try to improve the data analysis. It is designed to track through crossing fibers by also considering the secondary diffusion direction. (Behrens, Berg, Jbabdi, Rushworth, \& Woolrich, 2007). However, due to the limitation of the current DTI technology, the anatomical architecture of the language pathways are not precisely established yet. There are still debates on the location or end points of some language fibers (Bernal \& Ardila, 2009).

Another point to note is that the subjects in Sample 2 were scanned using more advanced protocols than those in to Sample 1. This is because the data for Sample 2 was acquired later than for Sample 1, and updates to the currently most advanced scanning protocols in our institute have taken place. Also, due to the high costs of imaging, the sample size of studies such as the present one, is usually kept relatively small.

As the first of its kind, the research reported here was exploratory in nature. Given the above caveats, we look forward to future replications and extensions in order to be able to draw any firm conclusions.

\section{Note}

1 This measure of IQ was used in preference to other, more comprehensive alternatives such as WAIS in this study due to severe time constraints. As a reviewer notes, though, the WAIS III's Verbal IQ scale would be an interesting choice for future work, because of its focus on verbal intelligence, rather than non-verbal intelligence as is the case for the Raven test. 


\section{References}

Alexander, A. L., Hasan, K. M., Lazar, M., Tsuruda, J. S., \& Parker, D. L. (2001). Analysis of partial volume effects in diffusion-tensor MRI. Magnetic Resonance in Medicine, 45, 770-780.

Basser, P. J., \& Pierpaoli, C. (1996). Microstructural and physiological features of tissues elucidated by quantitative-diffusion-tensor MRI. Journal of Magnetic Resonance B, 111, 209-219.

Behrens, T. E., Woolrich, M. W., Jenkinson, M., Johansen-Berg, H., Nunes, R. G., Clare, S., et al. (2003). Characterization and propagation of uncertainty in diffusion-weighted MR imaging. Magnetic Resonance Med, 50, 1077-1088.

Behrens, T. E. J., Berg, H. J., Jbabdi, S., Rushworth, M. F. S., \& Woolrich, M. W. (2007). Probabilistic diffusion tractography with multiple fiber orientations: What can we gain? Neuroimage, 34, 144-155.

Bernal, B., \& Ardila, A. (2009). The role of the arcuate fasciculus in conduction aphasia. Brain, 132, 2309-2316.

Bookheimer, S. (2002). Functional MRI of language: New approaches to understanding the cortical organization of semantic processing. Annual Review of Neuroscience, 25, 151-188.

Bullmore, E., Owen, A. M., McMillan, K. M., \& Laird, A. R. (2005). N-back working memory paradigm: A meta-analysis of normative functional neuroimaging. Human Brain Mapping, 25, 46-59.

Catani, M., \& de Schotten, M. T. (2008). A diffusion tensor imaging tractography atlas for virtual in vivo dissections. Cortex, 44, 1105-1132.

Catani, M., Allin, M. P., Husain, M., Pugliese, L., Mesulam, M. M., Murray, R. M., et al. (2007). Symmetries in human brain language pathways correlate with verbal recall. Proc Natl Acad Sci US A, 104, 17163-17168.

Catani, M., Howard, R. J., Pajevic, S., \& Jones, D. K. (2002). Virtual in vivo interactive dissection of white matter fasciculi in the human brain. Neuroimage, 17, 77-94.

D’Esposito, M., Aguirre, G. K., Zarahn, E., Ballard, D., Shin, R. K., \& Lease, J. (1998). Functional MRI studies of spatial and nonspatial working memory.

Cognitive Brain Research, 7, 1-13.

Deary, I. J., Penke, L., \& Johnson, W. (2010). The neuroscience of human intelligence differences. Nature Reviews Neuroscience, 11, 201-211.

Friederici, A. D., Bahlmann, J., \& Schubotz, R. I. (2008). Hierarchical artificial grammar processing engages Broca's area. Neuroimage, 42, 525-534.

Friederici, A. D. (2009). Pathways to language: Fiber tracts in the human brain. Trends in Cogntive Science (Regul. Ed.), 13, 175-181.

Hagoort, P. (2005). On Broca, brain, and binding: A new framework. Trends in Cognitive Science, 9, 416-423. 
Hamel, R., \& Schmittmann, V. D. (2006). The 20-minute version as a predictor of the Raven Advanced Progressive Matrices Test. Educational and Psychological Measurement, 66, 1039-1046.

Hua, K., Zhang, J., Wakana, S., Jiang, H., Li, X., Reich, D. S., et al. (2008). Tract probability maps in stereotaxic spaces: Analyses of white matter anatomy and tract-specific quantification. Neuroimage, 39, 336-347.

Jung, R. E., \& Haier, R. J. (2007). The Parieto-Frontal Integration Theory (P-FIT) of intelligence: Converging neuroimaging evidence. Behavioral and Brain Sciences, 30, 135-154.

Le Bihan, D., Mangin, J. F., Poupon, C., Clark, C. A., Pappata, S., Molko, N., et al. (2001). Diffusion tensor imaging: Concepts and applications. Journal of Magnetic Resonance Imaging, 13, 534-546.

Lebel, C., \& Beaulieu, C. (2009). Lateralization of the arcuate fasciculus from childhood to adulthood and its relation to cognitive abilities in children. Humam Brain Mapping, 30, 3563-3573.

Liederman, J., Gilbert, K., Fisher, J. M., Mathews, G., Frye, R. E., \& Joshi, P. (2011). Are women more influenced than men by top-down semantic information when listening to disrupted speech? Lang Speech, 54, 33-48.

Makris, N., \& Pandya, D. N. (2009). The extreme capsule in humans and rethinking of the language circuitry. Brain Structure \& Function, 213, 343-358.

Makris, N., Kennedy, D. N., McInerney, S., Sorensen, A. G., Wang, R., Caviness, V. S., et al. (2005). Segmentation of subcomponents within the superior longitudinal fascicle in humans: A quantitative, in vivo, DT-MRI study. Cerebral Cortex, 15, 854-869.

Mechelli, A., Crinion, J. T., Noppeney, U., O’Doherty, J., Ashburner, J., Frackowiak, R. S., et al. (2004). Neurolinguistics: Structural plasticity in the bilingual brain. Nature, 431, 757.

Meara, P. (2005). Llama Language Aptitude Tests. The manual. Swansea, UK: University of Wales.

Musso, M., Moro, A., Glauche, V., Rijntjes, M., Reichenbach, J., Buchel, C., et al. (2003). Broca's area and the language instinct. Nature Neuroscience, 6, 774-781.

Poldrack, R. A., Wagner, A. D., Prull, M. W., Desmond, J. E., Glover, G. H., \& Gabrieli, J. D. E. (1999). Functional specialization for semantic and phonological processing in the left inferior prefrontal cortex. Neuroimage, 10, 15-35.

Price, C. J., Lee, H., Devlin, J. T., Shakeshaft, C., Stewart, L. H., Brennan, A., et al. (2007). Anatomical traces of vocabulary acquisition in the adolescent brain. Journal of Neuroscience, 27, 1184-1189.

Richardson, F. M., \& Price, C. J. (2009). Structural MRI studies of language function in the undamaged brain. Brain Structure \& Function, 213, 511-523.

Schultz, T., \& Seidel, H. P. (2008). Estimating crossing fibers: A tensor decomposition approach. IEEE Trans Vis Comput Graph, 14, 1635-42.

Shaw, P. (2007). Intelligence and the developing human brain. Bioessays, 29, 962-973. 
Smith, E. E., \& Jonides, J. (1998). Neuroimaging analyses of human working memory. Proceedings of the National Academy of Sciences of the United States of America, 95, 12061-12068.

Thompson, P. M., Chiang, M. C., Barysheva, M., Shattuck, D. W., Lee, A. D., Madsen, S. K., et al. (2009). Genetics of brain fiber architecture and intellectual performance. Journal of Neuroscience, 29, 2212-2224.

Turken, A. U., Whitfield-Gabrieli, S., Bammer, R., Baldo, J. V., Dronkers, N. F., \& Gabrieli, J. D. E. (2008). Cognitive processing speed and the structure of white matter pathways: Convergent evidence from normal variation and lesion studies. Neuroimage, 42, 1032-1044.

Tzourio-Mazoyer, N., Landeau, B., Papathanassiou, D., Crivello, F., Etard, O., Delcroix, N., et al. (2002). Automated anatomical labeling of activations in SPM using a macroscopic anatomical parcellation of the MNI MRI single-subject brain. Neuroimage, 15, 273-289.

van den Noort, M., Bosch, P., Haverkort, M., \& Hugdahl, K. (2008). A standard computerized version of the reading span test in different languages. European Journal of Psychological Assessment, 24, 35-42.

Wechsler, D. (1997). Wechsler memory scale-Third edition. San Antonio, TX: The Psychological Corporation.

Westerhausen, R., Helland, T., Ofte, S., \& Hugdahl, K. (2010). A longitudinal study of the effect of voicing on the dichotic listening ear advantage in boys and girls at age 5 to 8. Developmental Neuropsychology, 35, 752-761.

Xiang, H. D., Fonteijn, H. M., Norris, D. G., \& Hagoort, P. (2010). Topographical functional connectivity pattern in the perisylvian language networks. Cereb Cortex, 20, 549-560.

Xiang, H. D., van Leeuwen, T. M., Dediu, D., Roberts, L., Norris, D. N., Hagoort, P. (submitted). L2-proficiency-dependent laterality of structural connectivity on brain language pathways. Brain and Language.

Yu, A. C. L. (2010). Perceptual compensation is correlated with individuals' "autistic" traits: Implications for models of sound change. PLoS One, 5.

Zweier, C., de Jong, E. K., Zweier, M., Orrico, A., Ousager, L. B., Collins, A. L., et al. (2009). CNTNAP2 and NRXN1 are mutated in autosomal-recessive Pitt-Hopkins-like mental retardation and determine the level of a common synaptic protein in Drosophila. American Journal of Human Genetics, 85, 655-666. 


\section{Appendix}

The language background of the participants

\begin{tabular}{|c|c|c|c|}
\hline & \multicolumn{3}{|c|}{$\begin{array}{c}\text { Language/Starting-age (years)/ } \\
\text { Way-of-learning/Total-time-of-learning (years) }\end{array}$} \\
\hline & $1^{\text {st }}$ language & $2^{\text {nd }}$ language & $3^{\text {rd }}$ language \\
\hline p01 & German/0/both/22 & English/11/formal/11 & French/13/formal/7 \\
\hline p05 & German/0/both/20 & English/10/both/9 & Spanish/17/both/3 \\
\hline p06 & German/0/both/19 & English $/ 11 /$ formal $/ 8$ & \\
\hline p07 & German/0/both/21 & English/10/formal/8 & French/13/formal/5 \\
\hline $\mathrm{p} 12$ & German/0/both/23 & English/10/formal/13 & Italian/15/informal/1 \\
\hline p14 & German/0/both/20 & English/10/formal/9 & Spanish/17/formal/2 \\
\hline p15 & German/0/both/20 & English/10/formal/10 & Spanish/17/formal/3 \\
\hline p16 & German/0/both/20 & English/8/formal/11 & Spanish/14/formal/2 \\
\hline p18 & German/0/both/20 & English/10/both/10 & \\
\hline p20 & German/0/both/19 & English/9/both/10 & French/12/both/10 \\
\hline p21 & German/0/both/20 & English/12/formal /8 & French/18/formal/2 \\
\hline p22 & German/0/both/21 & English/10/formal/10 & French/1/informal/1 \\
\hline p24 & German/0/both/21 & English/10/formal/10 & \\
\hline p25 & German/0/both/20 & English/9/both/11 & French/11/formal/4 \\
\hline p28 & German/0/both/19 & English/9/both/11 & French/15/formal/4 \\
\hline p30 & German/0/both/19 & English/9/both/11 & French/15/formal/4 \\
\hline p31 & Dutch/0/both/17 & English/12/both/7 & German/14/formal/4 \\
\hline p32 & German/0/both/19 & English/12/both/5 & Latin/14/formal/3 \\
\hline p33 & Dutch/0/both/22 & English/10/both/8 & Spanish/19/formal/1 \\
\hline p34 & Dutch/0/both/19 & German/4/both/19 & English/10/formal/9 \\
\hline p35 & Dutch/0/both/20 & English/6/both/14 & \\
\hline
\end{tabular}

Note. a) Since the information of the language background is acquired one year after the original experiment in responding to the reviewer's request, we did not manage to get all the participants back. We put all the info we have collected in this table, but not for all subjects. b) The "formal" learning specifically refers to the classroom learning, otherwise it is indicated as the "informal" learning. "Both" means both formal and informal learning have occurred. 